\title{
A generalized manifold topology for branching space-times
}

\author{
Thomas Müller*
}

January 4, 2013

\begin{abstract}
The logical theory of branching space-times (Belnap, Synthese 1992), which provides a relativistic framework for studying objective indeterminism, remains mostly disconnected from the discussion of space-time theories in the philosophy of physics. Earman has criticized the branching approach and suggested "pruning some branches from branching space-time" (2008).

This paper identifies the different - order-theoretic vs. topologicalpoints of view of both discussions as a reason for certain misunderstandings, and tries to remove them. Most importantly, we give a novel, topological criterion of modal consistency that usefully generalizes an earlier criterion, and we introduce a differentialgeometrical version of branching space-times as a non-Hausdorff (generalized) manifold.
\end{abstract}

\section{Introduction}

Discussions of determinism and indeterminism play an important role in many areas of philosophy. In philosophy of science, they have acted as probes into the basic structure of many physical theories; in philosophical logic, such discussions have triggered the development of a number of different logical systems, some of which have found applications in computer science.

\footnotetext{
*Department of Philosophy, Utrecht University, Janskerkhof 13a, 3512 BL Utrecht, The Netherlands; Thomas.Mueller@phil.uu.nl. This is a preprint of the paper presented at the PSA2012 Conference in San Diego, November 2012. The paper is forthcoming in Philosophy of Science. Copyright by the author.
} 
When it comes to defining indeterminism, one can discern two related, but technically different approaches. The basic idea behind indeterminism is that given the way things are at one moment in time, more than one future course of events is possible. One way to spell this out is to start with a class of separate possible courses of events and the notion of a state at a moment. If the same state occurs in at least two different courses of events that disagree about the future development after the respective occurrences, the class is indeterministic. If that class is given via a scientific theory, that theory is accordingly diagnosed to be indeterministic. As a way of classifying scientific theories, this approach was pioneered by Montague (1962). After several refinements (see, e.g., Earman (2006)), many of which were triggered by specific issues of space-time theories, this approach grounds the generally accepted definition of indeterminism for scientific theories: "a theory is deterministic if, and only if: for any two of its models, if they have instantaneous slices that are isomorphic, then the corresponding final segments are also isomorphic" (Butterfield, 2005).

A different way to model indeterminism is to start not with separate courses of events (called "models" in the above quote, but see §2) to be matched via their states at moments, but with a unified structure of moments within which one can identify the possible courses of events - histories as substructures. This approach is more in line with an indexical understanding of branching future possibilities, according to which different possible courses of events can literally share a past segment. Such branching history structures were developed in Prior's tense logic (Prior, 1967); an important early paper is Thomason (1970). In so-called "branching time" (BT; a somewhat misleading label, as time does not branch, only temporal histories do), a single history is pictured as a linearly ordered set of moments carved from a global partial ordering of moments. BT is used as a background for the "seeing to it that" logic of agency (Belnap et al., 2001) and arguably corresponds well to the phenomenology of an open future. The framework is however non-relativistic: a moment (an element of the ordering) has to represent all of space simultaneously.

Theories of branching space-times (BST) extend the branching histories idea by taking histories to be space-times rather than linearly ordered chains of moments. Belnap's version of BST (BST92; Belnap, 1992) provides a mathematically rigorous theory of objective indeterminism in a relativistic setting. BST92 retains BT's underlying algebraic approach of starting with a partially ordered set that can be called Our World as it represents all events that are, were or will be possible. The notion of modal consistency, i.e., possible co-occurrence within a single consistent course of events, which in the case of BT comes down to order-relatedness (linearity), has to be extended: In the case of BST92, consistency is taken to mean the existence of a common upper bound (two events are compatible if there is, so to speak, a perspective from which both events have occurred). Building on 
that definition, the histories in Our World are defined as maximally modally consistent subsets. These histories represent the possible courses of events in our world - complete space-times - that branch off from one another at choice points whose future light cones differentiate the histories.

Despite its relativistic soundness and some applications to physical theories (e.g., Placek, 2010), BST has not been much referenced in discussions of space-time theories, even when such discussions are concerned with questions of determinism and indeterminism. In fact, BST92 and some related BST theories have received a fair share of critique based on technical considerations of the space-time models of general relativity (Earman, 2008). Some of that criticism does not apply to BST92 since it only points out conceptual unclarities in relatives of that framework and not in BST92 itself, but there remains an important point of criticism in that BST92 corresponds poorly to general relativity's background in differential geometry. While some recent publications (e.g., Placek and Belnap, 2012) have begun to address this worry, we agree that so far, no extant formal specification of BST fully meets the critique.

This paper spells out an overarching framework that is meant to do justice both to the logical aspects of BST and to the physical considerations supporting general relativistic space-time theories. It will be proved that our framework, which deviates from the mentioned theory of BST92 in some technical details, is truly a theory of branching (in the sense of logic) spacetimes (in the sense of general relativity).

\section{Terminology}

When discussing formal models that incorporate modality, such as theories of branching histories, it is important to use terminology that allows to make some necessary distinctions. Modal consistency ("modal flatness") is usually a property of substructures and not of a full structure, which incorporates different alternatives and is therefore meant to be modally inconsistent. We suggest to distinguish:

- a logical theory, specified via a set of axioms in some formal language,

- models of a logical theory, i.e., structures fulfilling the axioms,

- a physical theory, which normally should not be identified with some axiomatic framework,

- a solution to the equations of the theory, which is a mathematical structure, and can often be identified with a history (a complete possible course of events), and

- the metaphysical notion of a world as something that is unified by "suitable external relations" (Lewis, 1986, 208). 
It is common to identify the notions of model, solution to the equations, history, and (possible) world. This identification is however not mandatory, and in fact positively harmful when it comes to BST. A history (possible course of events) indeed has to be "modally flat", containing no modally incompatible events. In the case of BST, models however contain more than one history, and each model (in the logical sense) specifies a world (in the given metaphysical sense). Following Belnap, we propose to call such a world (a model of BST) "Our World" rather than a "possible world", since the notion of a possible world tends to trigger the image of modal consistency (while, to repeat, this is not part of the definition of what a world is).

\section{$3 \quad$ An overview of BST92}

Here we give the axiomatic basis of BST92, due to Belnap (1992, 2003). Later on we will deviate from this framework in a small but substantial matter of topological detail in order to move it closer to general relativity.

A model of BST92 is a non-empty partial order $\langle W, \leq\rangle$ (a nonempty set $W$ together with a transitive, antisymmetric relation $\leq)^{1}$ subject to the set of constraints given below. Elements of $W$ are called possible point events, or, briefly, events. Let $H \subseteq \wp W$ be the set of maximal upward directed subsets of $W$. (In a partial order, a set is upward directed iff for any two of its elements $a$ and $b$, there is an element $c$ s.t. $a \leq c$ and $b \leq c$. We often shorten to "directed".) Elements of $H$, i.e., maximal directed subsets $h \subseteq W$, are called histories. A chain in $W$ is a linear subset, i.e., a subset $c \subseteq W$ s.t. for any $x, y \in c$ we have either $x \leq y$ or $y<x$.

The axioms of BST92 are as follows:

- $\langle W, \leq\rangle$ is a nonempty, dense partial order without maxima.

- Each lower bounded chain $C \subseteq W$ has an infimum in $W$, written inf $C$.

- Each upper bounded chain $C \subseteq W$ has a supremum-in- $h\left(\sup _{h} C\right)$ for each history $h \in H$ for which $C \subseteq h$.

- (Prior choice principle.) If $C \in h-h^{\prime}$ is a lower bounded chain in $h$ none of whose elements is an element of $h^{\prime}$, then there is a choice point $c \in h \cap h^{\prime}$ such that $c$ is maximal in $h \cap h^{\prime}$, and $c<C$ (i.e., for all $e \in C$, we have $c<e$ ).

Note that by the given definition, histories are downward closed: if $e \in h$ and $f \in W$ s.t. $f \leq e$, then also $f \in h$. Accordingly, if $c$ is a lower bounded chain in history $h$, then $\inf c \in h$ as well.

\footnotetext{
${ }^{1}$ We will also use the relation symbol " $<$ ", which is defined in the usual way: $x<y$ iff $(x \leq y$ and $x \neq y)$.
} 
As a first link with space-time theories, we can give a generic definition of the causal and the chronological past and future of events in a BST92 model, as follows:

Definition 1 Given a BST92 model $\langle W, \leq\rangle$, an event $e \in W$ lightlike precedes $f \in W$ (in symbols: $e \triangleleft f$ ) iff $e \leq f$ and there is only a single maximal chain that has $e$ as its first and $f$ as its last point. Event $e$ chronologically precedes $f$ (in symbols: $e \ll f$ ) iff $e<f$ and it is not the case that $e \triangleleft f$.

Based on these notions we can define the notions of the causal and the chronological future (and analogously, past) of an event $e \in W$, as usual:

Definition 2 Given a BST92 model $\langle W, \leq\rangle$ and some $e \in W$, the causal future of $e, J^{+}(e)$, and the chronological future, $I^{+}(e)$, are defined as follows:

$$
J^{+}(e):=\{f \in W \mid e \leq f\} ; \quad I^{+}(e):=\{f \in W \mid e \ll f\} .
$$

The corresponding past notions are:

$$
J^{-}(e):=\{f \in W \mid f \leq e\} ; \quad I^{-}(e):=\{f \in W \mid f \ll e\} .
$$

\section{The Hausdorff property in space-time theories}

A topological space $\langle X, \mathcal{R}\rangle$, where $X$ is a nonempty set and the topology $\mathcal{R} \subseteq \wp X$ is the collecion of open sets, is Hausdorff if any two elements of $X$ can be separated by disjoint open sets. ${ }^{2}$ Hausdorffness forbids, intuitively speaking, the existence of "unseparably close points", or perhaps "doubled points" or "points that occupy the same position". The branching real line pictured in Fig. 1 is a simple example of a non-Hausdorff space. Following Hajicek (1971), who credits Geroch for the notation, we will write $x \mathrm{Y} y$ to indicate that the points $x$ and $y$ violate the Hausdorff condition, i.e., that $x$ and $y$ cannot be separated by disjoint open sets. In Fig. 1 we have $0_{1} \mathrm{Y}_{2}$. The notation usefully suggests graphically that in such a case, $x$ and $y$ "branch off" from some common trunk, like the left part of the branching lines of Fig. 1. In fact, in our examples below such $x$ and $y$ will always be different limits of a single converging sequence.

As defined, BST92 does not come with a topology. One natural topology has recently been discussed extensively by Placek and Belnap (2012). With respect to this topology, the Hausdorff property generically fails in models of BST92. Models of BST92 are, however, not locally Euclidean and thus do not form (generalized) manifolds. ${ }^{3}$

\footnotetext{
${ }^{2}$ For a simple introduction to relevant formal definitions and background, see, e.g., Mendelson (1990).

${ }^{3} \mathrm{~A}$ manifold is, roughly, a topological space that is locally Euclidean and that, therefore, locally "looks like" Euclidean space of a specific dimension. Malament (2012) gives a nice introduction to this and other notions from differential geometry. Normally, manifolds are required to be Hausdorff. A generalized manifold is allowed to be non-Hausdorff.
} 


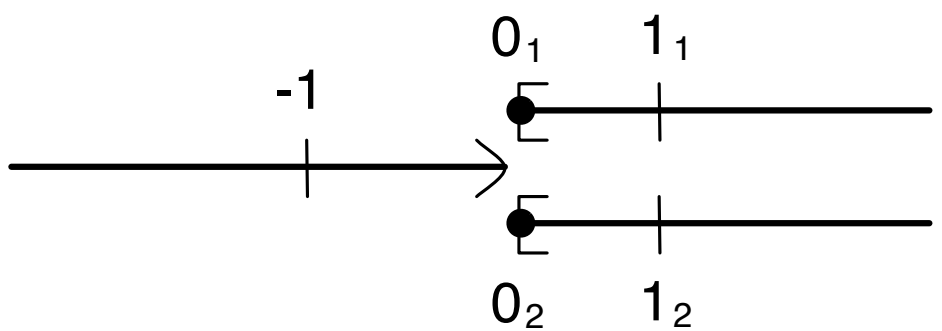

Figure 1: The branching real line as a simple non-Hausdorff space. A basis for the topology is given by the open intervals in both tracks, reaching into the common part on the left.

Is non-Hausdorffness a good idea for grounding BST? The discussion of this issue is somewhat tangled. Earman (2008) in his overview of "individual branching" for single space-times concludes that the only viable path to individually branching space-times comes from non-Hausdorff models, because other approaches (e.g., so-called trousers worlds) appear unfeasible. While we follow Earman in this assessment, we want to stress that BST - at least in the form of BST92 and in the form that we are trying to develop further here - is meant to give not a picture of a single space-time that somehow branches (perhaps like an amoeba undergoing fission), but to integrate several histories within one logical model. The branching (overlapping) histories are individually non-branching and in fact Hausdorff space-times. Still, it is useful to look at the physicists' discussion of non-Hausdorffness that Earman references.

In mathematical physics, Hajicek (1971) proves an important result about solutions to the Einstein field equations whose maximal analytic extensions are non-Hausdorff: Roughly, a non-Hausdorff space-time either fails to be strongly causal, or it admits bifurcating geodesics. He interprets this result as showing that "all such [i.e., non-Hausdorff] space-times must be weakly acausal" (Hajicek, 1971, 75), which would indeed be reason enough for a physicist to shun non-Hausdorff space-times. It is interesting to see how Hajicek supports his interpretation of his theorem. Commenting on bifurcating curves, he writes:

It is easily seen that such curves can only exist in a non-Hausdorff space. Then, if we have some system of ordinary differential equations which has locally a unique solution [...] it is immediate that this system cannot have two different solutions [...] unless these solutions form a bifurcate curve. Therefore, in view of the classical causality conception coinciding with determinism it is sensible to rule out the bifurcate curves. (Hajicek, 1971, 79) 
The dialectics is thus as follows: A result from mathematical physics (Hajicek's Theorem 4) establishes (roughly) that in non-Hausdorff space-time models there is either a violation of strong causality, or there are bifurcating curves. An appeal to determinism rules out the latter; considerations of physicality rule out the former. This amounts to rejecting non-Hausdorff models.

We agree with this argument completely. If BST were to give models of a single space-time, these models should not contain bifurcating curves, and most probably they shouldn't be weakly acausal either, so that nonHausdorffness would be ruled out. If one however takes up the issue of non-Hausdorff models in order to build formal models for indeterminism, which is what drives the development of BST, then the above argument obviously pulls no weight. The main challenge for bringing together BST and general relativity, in our view, lies not in non-Hausdorffness, but in the failure of BST92 to provide generalized manifolds. ${ }^{4}$

\section{Simple branching: generalized Minkowskian mani- folds}

We move on to the construction of simple branching models that provide a bridge between the logical, order-theoretic point of view of BST (histories, i.e., single space-times, as directed sets) and the topological point of view of general relativity (a single space-time as a differential manifold) ${ }^{5}$

The main technical challenge is to define structures in which two or more space-times are pasted together in such a way that the resulting object is locally Euclidean and makes sense as a model of objective indeterminism. In this paper we discuss the simplest case of such pasting, which is to paste together $m$ Minkowski space-times (of dimension $n>1$ ) at the origin, to arrive at a structure $M_{m}^{n}$. There are various choices for this pasting, depending on whether the $m$ origins are represented by one or by $m$ different points in the resulting structure, and on how the rim of the future light cone at the origin is handled. BST92's prior choice principle demands to identify the $m$ origins as one point, but to keep separate the rim of the future light cones above the origin. This is what breaks local Euclidicity: on such a structure, one cannot define a locally Euclidean topology (unless one gives up connectedness, which is not an interesting option). Since we want to

\footnotetext{
${ }^{4}$ We hereby follow Earman $(2008,198 \mathrm{f}$.): "topological spaces that are not locally Euclidean cannot be assigned a differentiable structure, and such a structure is essential in formulating the very notion of a Lorentzian metric and in formulating the Einstein field equations". Thus, if we want to remain close to general relativity, we had better arrive at a generalized manifold.

${ }^{5}$ For reasons of space, the following discussion is quite compressed. For a more detailed exposition, see Müller (2011).
} 
define generalized manifolds, we have to ascertain local Euclidicity. This means that we need to differ from BST92 in the pasting construction.

Our choice is to define structures of $m$-fold branching, $n$-dimensional Minkowski space-time $M_{m}^{n}$ as follows: ${ }^{6}$

Definition $3\left(M_{m}^{n}\right)$ The $m$-fold branching, $n$-dimensional Minkowski spacetime $M_{m}^{n}$ is defined from the $n$-dimensional Minkowski space-time $M^{n}$ (for simplicity, we use $\mathbb{R}^{n}$ with the Minkowskian ordering $\leq_{M}$ ) by setting a tobe-multiplied region $V$ to be the future light cone of the origin, including the rim of the light cone and the origin itself:

$$
V:=J^{+}(\boldsymbol{O})=\left\{\boldsymbol{x} \in M^{n} \mid \boldsymbol{O} \leq_{M} \boldsymbol{x}\right\} ; \quad \bar{V}:=M^{n}-V ; \quad V_{i}:=V \times\{i\} ;
$$

defining $m$ layers, for $i=1, \ldots, m$, to be

$$
L_{i}^{n}:=(\bar{V} \times\{1\}) \cup V_{i}
$$

and pasting them via

$$
M_{m}^{n}:=\bigcup_{i=1}^{m} L_{i}^{n} .
$$

The ordering $\leq$ on $M_{m}^{n}$ is the union of the usual Minkowskian orderings in the layers, and the locally Euclidean topology $\mathcal{R}$ is given via the countable basis of open balls with rational center coordinates $\boldsymbol{x}$ and rational radius $r$ in the finitely many layers $i=1, \ldots, m$,

$$
B_{i}(\boldsymbol{x}, r):=\left\{\langle\boldsymbol{y}, j\rangle \in L_{i}^{n} \mid d(\boldsymbol{x}, \boldsymbol{y})<r \& j \in\{1, i\}\right\} .
$$

Note that the layers themselves, each of which is homeomorphic to $\mathbb{R}^{n}$, are open sets in this topology, and that for $i \neq j, L_{i}^{n}-L_{j}^{n}=V_{i}$. Note also that $\partial V_{i}=\partial J^{+}(\mathbf{0}) \times\{i\}$.

\footnotetext{
${ }^{6}$ For a similar construction in the context of BST92, which however does not lead to generalized manifolds, see, e.g., Wroński and Placek (2009). An $M_{m}^{n}$-like construction is given in Visser (1996, 251-255); the book contains many pointers to relevant literature. Visser calls his construction a "branched spacetime" (252), without however making any connections to the philosophical/logical discussions about branching space-times. Penrose $(1979,593)$ has a suggestive drawing of a branching space-time; while Penrose is not explicit about the topology, and his Figure 12.3(a) may suggest choice points à la BST92, he seems to have our option in mind as well, since he writes: "on each branch the wavefunction starts out as a different eigenvector ..." (Penrose, 1979, 594; italics mine). Deutsch (1991) refers to this discussion; his remarks about "a larger object which has yet to be given a proper geometrical description" (3207) may be read as pointing in the direction of something like our $M_{m}^{n}$ structures, or their generalizations mentioned below. McCabe (2005) reproduces Penrose's figure. He remarks that such figures themselves are open to different interpretations and do not need to be read as implying non-Hausdorffness; this is in line with our view that there are in fact multiple options for pasting. However, McCabe does not discuss in much detail the price that has to be paid for dropping local Euclidicity in avoiding non-Hausdorffness, remarking that "it is a debate which has not been conducted in the literature" (McCabe, 2005, 670). We agree with Earman that local Euclidicity has to be taken very seriously, and we will continue to hold on to it.
} 


\section{Capturing modal consistency}

Our structures $M_{m}^{n}$ are still partial orders, as in BST92, but we want to move from the order-theoretic to a generalizable, topological characterization of modal consistency. Intuitively and by the pasting construction, the maximal modally consistent subsets of $M_{m}^{n}$ should be exactly the layers $L_{i}^{n}, i=$ $1, \ldots, m$. These cover the whole of $M_{m}^{n}$ without any gaps or holes, and they are also individually such that in each layer, each space-time point of Minkowski space-time $M^{n}$ occurs exactly once. These layers are also the histories in the sense of the usual, order-theoretic definition of BST92: each layer is a maximal directed set in $M_{m}^{n} \cdot{ }^{7}$ The question before us is how to capture this intuitive notion of modal consistency in purely topological terms.

Hajicek (1971) defines the useful notion of an $H$-submanifold of a $\mathrm{Y}$ manifold (where the " $H$ " stands for "Hausdorff", "Y" graphically represents non-Hausdorffness as branching, and a $\mathrm{Y}$-manifold is a generalized manifold):

Definition 4 (H-manifold) Given a $Y$-manifold $M$, a subset $A \subseteq M$ is an $H$-submanifold iff $A$ is open, connected, Hausdorff, and maximal with respect to these properties. (I.e., every proper superset of $A$ is either not open, not connected, or not Hausdorff.)

Hajicek (1971) also suggests to write $\mathrm{Y}_{M}^{L}$ for the set of points in $M$ that are non-Hausdorff related to some point in $L$,

$$
\mathrm{Y}_{M}^{L}:=\{x \in M \mid \exists y \in L x Y y\} .
$$

On our way towards a useful generalized notion of modal consistency, we note some facts about the points in $M_{m}^{n}$ that are non-Hausdorff related to some other point (obviously there are no such points in case $m=1$ ):

Lemma 1 Let $M:=M_{m}^{n}$ for some $n \in \mathbb{N}$ and some $m \geq 2$. Then for $x=\langle\boldsymbol{x}, i\rangle, y=\langle\boldsymbol{y}, j\rangle \in M$ we have

$$
x Y y \quad \text { iff } \quad \boldsymbol{x}=\boldsymbol{y}, i \neq j, \text { and } \boldsymbol{x} \in \partial J^{+}(\boldsymbol{O}) .
$$

Accordingly,

$$
Y_{M}^{M}=\left\{\langle\boldsymbol{x}, i\rangle \mid \boldsymbol{x} \in \partial J^{+}(\boldsymbol{O}) \& i \in\{1, \ldots, m\}\right\},
$$

and for $L:=L_{i}^{n}$ a layer $(i \in\{1, \ldots, m\})$, we have

$$
Y_{M}^{L}=\left\{\langle\boldsymbol{x}, j\rangle \mid \boldsymbol{x} \in \partial J^{+}(\boldsymbol{O}) \& j \in\{1, \ldots, m\} \& j \neq i\right\} .
$$

\footnotetext{
${ }^{7}$ Obviously the layers are directed sets, being order isomorphic to $M^{n}$, which is directed. For maximality, observe that any "new" element to be added to $L_{i}^{n}$ has to come from $V_{j}$ with $j \neq i$; by the definition of the ordering, the resulting superset of $L_{i}^{n}$ is not directed.
} 
Using a further lemma, we can then prove that the layers of $M_{m}^{n}$ are in fact $H$-submanifolds: ${ }^{8}$

Lemma 2 Let $M=M_{m}^{n}$ for some $n, m$, and let $L:=L_{i}^{n} \subseteq M$ be a layer $(i \in\{1, \ldots, m\})$. Then $L$ is an $H$-submanifold of $M$.

However, the notion of an $H$-submanifold is not sufficient as an analysis of modal consistency, given that we also want the other direction of Lemma 2:

Fact $1 M:=M_{2}^{2}$ has an $H$-submanifold that is not equal to one of the layers $L_{i}^{n}, i=1,2$.

Proof by example: We divide the rim of the forward light-cone of the origin into a left and a right part, which are allowed to overlap at the origin:

$$
J_{l}:=\left\{\langle t, x\rangle \in J^{+}(\mathbf{0}) \mid x \leq 0\right\}, J_{r}:=\left\{\langle t, x\rangle \in J^{+}(\mathbf{0}) \mid x \geq 0\right\} .
$$

We have $J_{l} \cup J_{r}=J^{+}(\mathbf{0})$ and $J_{l} \cap J_{r}=\{\mathbf{0}\}$. Now consider the set

$$
A:=M-\left(\left(J_{l} \times\{1\}\right) \cup\left(J_{r} \times\{2\}\right)\right),
$$

i.e., $A$ is the whole of the pasted space $M$ without half of the rim of the forward light-cone in each layer. Note that the origin in both layers is removed in constructing $A$, which makes it intuitively weird. But as a fact, $A$ is a $H$-submanifold of $M$. This follows directly from Hajicek (1971, Theorem 2). So, we know that not every $H$-submanifold can be taken to be a history.

By a more detailed consideration of this and other examples, we are finally led to our official topological definition of a maximal consistent set, or a history: ${ }^{9}$

Definition 5 Given $M=M_{m}^{n}$ for some $n$ and $m$, $a$ history in $M$ is a subset $h \subseteq M$ that is maximal with respect to the properties of being (i) open, (ii) connected, (iii) Hausdorff, and (iv) for each subset $C \subseteq h$, if $\partial C \neq \emptyset$, then $h \cap \partial C \neq \emptyset$ as well.

As a test for the usefulness of this definition, we can now indeed prove both directions of the analogue of Lemma 2:

Lemma 3 Given $M=M_{m}^{n}$ for some $n$ and $m$, a subset $A \subseteq M$ is a history according to Definition 5 iff $A=L_{i}^{n}$ for some $i \in\{1, \ldots, m\}$.

Definition 5 can be applied to any $H$-manifold, it is not limited to the structures $M_{m}^{n}$ with respect to which it was motivated. So we have arrived at a general definition of modal consistency in branching space-times.

\footnotetext{
${ }^{8}$ For details, see Müller (2011).

${ }^{9}$ For details, see again Müller (2011).
} 


\section{Conclusion}

In this paper we have given an overview of the main challenges facing the construction of explicit formal models for indeterminism in a general relativistic setting. The best extant candidate framework for such models is Belnap's BST92. Some criticisms leveled against the project of constructing branching space-times seem misplaced as they rely on the idea that in BST, a single space-time should somehow branch or bifurcate. We agree that this should be avoided. The branching in BST is of a modal nature, and as single space-times are modally consistent, they themselves do not branch. The BST approach, once it is followed with mathematical rigor, and once a topology is defined, does however lead to non-Hausdorff models. Again, a fair share of the criticism of non-Hausdorff models only applies to single space-times and not to BST structures. There is however one specific challenge that has so far not been met: BST92 does not define generalized manifolds, and therefore the link with general relativity is not satisfactory. We believe that we have met this challenge, at least for simple structures.

In this paper we have defined a method for pasting Minkowski spacetimes in a locally Euclidean way, such that the resulting structures are generalized manifolds. We have also given a novel definition of modal consistency, which is purely topological and which generalizes the order-theoretic definition in terms of directedness on which BST92 is built.

A lot remains to be done. So far we have only investigated the simplest structures: pasted Minkowski space-times. We propose that locally, any useful branching space-time should look like one of the $M_{m}^{n}$ we have defined here. The study of global features of our BST models, however, has to be left for future work.

\section{Acknowledgements}

Research leading to these results has received funding from the European Research Council under the European Community's Seventh Framework Programme (FP7/2007-2013) / ERC Grant agreement nr 263227, and from the Dutch Organization for Scientific Research, grant nr NWO VIDI 27620-013. Thanks to Nuel Belnap and Tomasz Placek for many stimulating discussions.

\section{References}

Belnap, N. (1992). Branching space-time. Synthese, 92(3):385-434. See also the postprint 2003, available on philsci-archive.

Belnap, N. (2003). No-common-cause EPR-like funny business in branching space-times. Philosophical Studies, 114:199-221. 
Belnap, N., Perloff, M., and Xu, M. (2001). Facing the future. Agents and choices in our indeterminist world. Oxford: Oxford University Press.

Butterfield, J. (2005). Determinism and indeterminism. In Routledge Encyclopedia of Philosophy, volume 3. London: Routledge.

Deutsch, D. (1991). Quantum mechanics near closed timelike lines. Physical Review D, 44(10):3197-3217.

Earman, J. (2006). Aspects of determinism in modern physics. In Butterfield, J. and Earman, J., editors, Handbook of the Philosophy of Physics, pages 1369-1434. Amsterdam: Elsevier.

Earman, J. (2008). Pruning some branches from branching space-times. In Dieks, D., editor, The Ontology of Spacetime II, pages 187-206. Amsterdam: Elsevier.

Hajicek, P. (1971). Causality in non-Hausdorff space-times. Communications in Mathematical Physics, 21:75-84.

Lewis, D. K. (1986). On the plurality of worlds. Oxford: Blackwell.

Malament, D. (2012). Topics in the Foundations of General Relativity and Newtonian Gravitation Theory. University of Chicago Press, Chicago IL.

McCabe, G. (2005). The topology of branching universes. Foundations of Physics Letters, 18(7):665-676.

Mendelson, B. (1990). Introduction to Topology. Dover, New York, NY, 3rd edition.

Montague, R. (1962). Deterministic theories. In Willner, D., editor, Decisions, Values and Groups, pages 325-370. Oxford: Pergamon Press. Reprinted in Formal Philosophy, ed. R.H. Thomason, New Haven, CT: Yale University Press 1974, pp. 303-359.

Müller, T. (2011). Branching space-times, general relativity, the Hausdorff property, and modal consistency. Technical report, Theoretical Philosophy Unit, Utrecht University. URL = http://philsci-archive.pitt.edu/8577/.

Penrose, R. (1979). Singularities and time-asymmetry. In Hawking, S. W. and Israel, W., editors, General relativity: an Einstein centenary survey, pages 581-638. Cambridge: Cambridge University Press.

Placek, T. (2010). Bell-type correlations in branching space-times. In Czarnecki, T., Kijania-Placek, K., Poller, O., and Woleński, J., editors, The Analytic Way. Proceedings of the 6th European Congres of Analytic Philosophy, pages 105-144. London: College Publications. 
Placek, T. and Belnap, N. (2012). Indeterminism is a modal notion: branching spacetimes and Earman's pruning. Synthese, 187(2):441-469. DOI: 10.1007/s11229-010-9846-8.

Prior, A. N. (1967). Past, Present and Future. Oxford: Oxford University Press.

Thomason, R. H. (1970). Indeterminist time and truth-value gaps. Theoria, $36: 264-281$.

Visser, M. (1996). Lorentzian Wormholes. New York, NY: American Institute of Physics.

Wroński, L. and Placek, T. (2009). On Minkowskian branching structures. Studies in History and Philosophy of Modern Physics, 40:251-258. 